VOL. 61 (2000) [335-343]

\title{
A FORMULA FOR THE EULER CHARACTERISTIC OF LINE SINGULARITIES ON SINGULAR SPACES
}

\author{
GuANGFEng JiAng
}

\begin{abstract}
We prove an algebraic formula for the Euler characteristic of the Milnor fibres of functions with critical locus a smooth curve on a space which is a weighted homogeneous complete intersection with isolated singularity.
\end{abstract}

\section{INTRODUCTION}

For an analytic function germ $f:(X, 0) \longrightarrow(\mathbb{C}, 0)$ with critical locus $\Sigma \subset X$, there is a local Milnor fibration induced by $f$. We are interested in the topology of the Milnor fibre $F$ of $f$ in the case when $\operatorname{dim} \Sigma=1$. It is well known that in this case the homotopy type of $F$ is not necessarily a bouquet of spheres in the middle dimension. The calculation of the Euler characteristic $\chi(F)$ of $F$ is of importance. There is a so called Iomdin-Lê formula [6] which expresses the Euler characteristic of the Milnor fibre of $f$ by that of the series of $f$ with isolated singularities.

The question we are interested in is if there is a way to express $\chi(F)$ by some "computable" invariants determined only by $(f, \Sigma, X)$. When $X$ is $\mathbb{C}^{m}$, the singular locus $\Sigma$ of $f$ is a one dimensional complete intersection with isolated singularity, and the transversal singularity type of $f$ along $\Sigma$ is Morse, Pellikaan [9] answered this question positively. Pellikaan's formula expresses the Euler characteristic in terms of the Jacobian number $j(f), \delta$ and the Milnor number $\mu(\Sigma)$ of $\Sigma$. These numbers can be computed directly by counting the dimensions of certain finite dimensional vector spaces. The development of computer algebra makes this kind of algebraic formulae more and more important and popular. In this article we answer the question by proving a similar formula for function germs with line singularities on a weighted homogeneous space $X$ with isolated complete intersection singularity (see Proposition 7). Note that for a function germ $f$ with isolated singularity on a weighted homogeneous complete intersection with isolated singularity, Bruce and Robert [1] have proved an algebraic formula for the Milnor number of $f$.

Received 22nd July, 1999

This article comes from part of the author's thesis which was completed at Utrecht University advised by Professor D. Siersma, to whom this author is indebted very much for many discussions, questions and remarks. This work was partially supported by JSPS, NNSFC, STCLN.

Copyright Clearance Centre, Inc. Serial-fee code: 0004-9727/00 \$A2.00+0.00. 


\section{NON-ISOLATED SINGULARITIES ON SINGULAR SPACES}

Let $\mathcal{O}_{\mathbb{C}^{m}}$ be the structure sheaf of $\mathbb{C}^{m}$. The stalk $\mathcal{O}_{\mathbb{C}^{m}, 0}$ of $\mathcal{O}_{\mathbb{C}^{m}}$ at 0 is a local ring, consisting of germs at 0 of analytic functions on $\mathbb{C}^{m}$. The ring $\mathcal{O}_{\mathbb{C}^{m}, 0}$ is often denoted by $\mathcal{O}_{m}$, or simply by $\mathcal{O}$ when no confusion can be caused. The unique maximal ideal of $\mathcal{O}_{m}$ is denoted by $\mathrm{m}_{m}$ or $\mathrm{m}$.

Let $(X, 0) \subset\left(\mathbb{C}^{m}, 0\right)$ be the germ of a reduced analytic subspace $X$ of $\mathbb{C}^{m}$ defined by an ideal $\mathfrak{h}$ of $\mathcal{O}$, generated by $h_{1}, \ldots, h_{p} \in \mathcal{O}$. Let $\mathfrak{g}$ be the ideal generated by $g_{1}, \ldots, g_{n} \in \mathcal{O}$. The germ of the analytic space defined by $\mathfrak{g}$ at 0 is denoted by $(\Sigma, 0)$. Write $\mathcal{O}_{X}:=\mathcal{O} / \mathfrak{h}$ and $\mathcal{O}_{\Sigma}:=\mathcal{O} / \mathfrak{g}$.

Let $\operatorname{Der}(\mathcal{O})$ denote the $\mathcal{O}$-module of germs of analytic vector fields on $\mathbb{C}^{m}$ at 0 . Then $\operatorname{Der}(\mathcal{O})$ is a free $\mathcal{O}$-module with $\frac{\partial}{\partial z_{1}}, \ldots, \frac{\partial}{\partial z_{m}}$ as basis, where $z_{1}, \ldots, z_{m}$ are the local coordinates of $\left(\mathbb{C}^{m}, 0\right)$. Der $(\mathcal{O})$ is a Lie algebra with the bracket defined by $[\xi, \eta]:=\xi \eta-\eta \xi$ for all $\xi, \eta \in \operatorname{Der}(\mathcal{O})$.

Define $\operatorname{Der}_{\mathfrak{h}}(\mathcal{O}):=\{\xi \in \operatorname{Der}(\mathcal{O}) \mid \xi(\mathfrak{h}) \subset \mathfrak{h}\}$, which is the $\mathcal{O}$-module of logarithmic vector fields along $(X, 0)$ and a Lie subalgebra of $\operatorname{Der}(\mathcal{O})[10,1]$. When $\mathfrak{h}$ is a radical ideal defining the analytic space $X, \operatorname{Der}_{\mathfrak{h}}(\mathcal{O})$ is often denoted by $D_{X}$. Geometrically, $D_{X}$ consists of all the germs of vector fields that are tangent to the smooth part of $X$. When $X$ is a weighted homogeneous complete intersection with isolated singularity, one can write down precisely all the generators of $D_{X}$ (see [13]).

For $f \in \mathcal{O}$, the ideal $J_{X}(f):=\left\{\xi(f) \mid \xi \in D_{X}\right\}$ is called the (relative) Jacobian ideal of $f$. Obviously, when $X$ is the whole space $\mathbb{C}^{m}$, namely, $\mathfrak{h}=\{0\}$, then $J_{X}(f)=J(f)$, the Jacobian ideal of $f$.

Let $\mathcal{S}=\left\{S_{\alpha}\right\}$ be an analytic stratification of $X, f:(X, 0) \longrightarrow(\mathbb{C}, 0)$ an analytic function germ. The critical locus $\Sigma_{f}^{S}$ of $f$ relative to the stratification $\mathcal{S}$ is the union of the critical loci of $f$ restricted to each of the strata $S_{\alpha}$, namely, $\Sigma_{f}^{S}=\bigcup_{S_{\alpha} \in \mathcal{S}} \overline{\Sigma_{f \mid S_{\alpha}}}$. We denote $\Sigma_{f}^{\mathcal{S}}$ by $\Sigma_{f}$ when $\mathcal{S}$ is clear from the context. If the dimension of $\Sigma_{f}^{\mathcal{S}}$ is not positive, we say that $f$ defines (or has) isolated singularities on $X$. If the dimension of $\Sigma_{f}^{S}$ is positive, we say that $f$ defines (or has) non-isolated singularities on $X$. If $\Sigma_{f}^{S}$ is a one dimensional smooth complex manifold, we say that $f$ defines (or has) a line singularity on $X$.

For an analytic space $X$ embedded in a neighbourhood $U$ of $0 \in \mathbb{C}^{m}$, there is a logarithmic stratification $\mathcal{S}_{\log }:=\left\{S_{\alpha}\right\}$ of $U$ (see $\left.[10,1]\right)$. In general, $\mathcal{S}_{\log }$ is not locally finite. If $\mathcal{S}_{\log }$ is locally finite, then $X$ is said to be holonomic.

Let $X$ be of pure dimension. The collection $\mathcal{S}_{\log }^{\prime}=\left\{X \cap S_{\alpha} \mid S_{\alpha} \in \mathcal{S}_{\text {log }}\right\}$ is a stratification of $X$ which will be called the logarithmic stratification of $X$ in this article. In particular, when $X$ has isolated singularity in 0 , then $\{0\}$ and the connected components of $X \backslash\{0\}$ form a holonomic logarithmic stratification of $X$. So 0 is always a critical point of any germ $f:(X, 0) \longrightarrow(\mathbb{C}, 0)$ relative to this stratification. Hence for $f \in \mathfrak{m}$, 
$\Sigma_{f}=\left\{p \in X \mid \xi(f)(p)=0\right.$ for all $\left.\xi \in D_{X}\right\}$ is the critical locus of $f$ relative to the logarithmic stratification. Obviously $\Sigma_{f}=X \cap \mathcal{V}\left(J_{X}(f)\right)$.

Definition 1. Let $\mathfrak{h}=\left(h_{1}, \ldots, h_{p}\right) \subset \mathfrak{g}=\left(g_{1}, \ldots, g_{n}\right)$ be ideals of $\mathcal{O}_{\mathbb{C}^{m}, 0}$. Define a subset of $\mathcal{O}$, called the primitive ideal of $\mathfrak{g}$ relative to $\mathfrak{h}$ :

$$
\int_{\mathfrak{h}} \mathfrak{g}:=\left\{f \in \mathfrak{g} \mid \xi(f) \subset \mathfrak{g} \text { for all } \xi \in \operatorname{Der}_{\mathfrak{h}}(\mathcal{O})\right\} .
$$

In the following we always assume that $\mathfrak{h}$ and $\mathfrak{g}$ are radical, $X=\mathcal{V}(\mathfrak{h})$ and $\Sigma=\mathcal{V}(\mathfrak{g})$. In this case, $\int_{\mathfrak{h}} \mathfrak{g}$ is denoted by $\int_{X} \mathfrak{g}$ or $\int \mathfrak{g}$ when no confusion can be caused by this. REMARKS 2.

(1) When $X$ is smooth this definition was given by Pellikaan $[7,8]$. It is straight forward to verify that $\int_{\mathfrak{h}} \mathfrak{g}$ is an ideal of $\mathcal{O}$, and $\mathfrak{g}^{2}+\mathfrak{h} \subset \int_{\mathfrak{h}} \mathfrak{g} \subset \mathfrak{g}$ always holds. And for $\mathfrak{g}_{i} \supset \mathfrak{h}(i=1,2)$, we have $\int_{\mathfrak{h}} \mathfrak{g}_{1} \cap \int_{\mathfrak{h}} \mathfrak{g}_{2}=\int_{\mathfrak{h}}\left(\mathfrak{g}_{1} \cap \mathfrak{g}_{2}\right)$;

(2) Geometrically, the relative primitive ideal collects all the functions whose zero level surfaces pass through $\Sigma$ and are tangent to the regular part $X_{\text {reg }}$ of $X$ along $\Sigma \cap X_{\text {reg. }}$.

(3) The singular locus (relative to $\mathcal{S}_{\log }^{\prime}$ ) of $f$ is $\Sigma_{f}:=\mathcal{V}\left(J_{X}(f)\right) \cap X$, and $\Sigma_{f} \subset f^{-1}(0)$ if $f(0)=0$. If $f \in \int \mathfrak{g}$, then $\Sigma \subset X \cap \mathcal{V}\left(J_{X}(f)\right)=\Sigma_{f}$. Conversely, for $f \in \mathfrak{m}$, we have $f \in \int \mathfrak{g}$ when $\Sigma \subset \Sigma_{f}$ and $\mathfrak{g}$ is radical. The reason is: $J_{X}(f) \subset \mathfrak{g}$, and since $f$ takes finite values on $\Sigma_{f}$ and $0 \in \Sigma$, $\left.f\right|_{\Sigma}=0$, so $f^{k} \in \mathfrak{g}$ for some $k \in \mathbb{N}$. Hence $f \in \mathfrak{g}$ since $\mathfrak{g}$ is radical.

(4) The relative primitive ideals have been generalised to higher relative primitive ideals in [5]. Under the assumption that $\mathfrak{h}$ is of pure dimension, $\mathfrak{g}$ is radical, and the Jacobian ideal of $\mathfrak{h}$ is not contained in any associated prime of $\mathfrak{g}$, it was proved that the primitive ideal $\int_{\mathfrak{h}} \mathfrak{g}$ is the inverse image in $\mathcal{O}$ of the second symbolic power of the quotient ideal $\overline{\mathfrak{g}}:=\mathfrak{g} / \mathfrak{h}$ of $\mathcal{O}_{X}$. Note that the results in $[5]$ generalise the results of $[11,7,8]$.

\section{Transversal singularities}

Let $(X, 0) \subset\left(\mathbb{C}^{n+1}, 0\right)$ be the germ of a reduced analytic space with isolated singularity in 0 . Let $\Sigma$ be a reduced curve germ on $(X, 0)$ defined by $g$ and have isolated singularity in 0 . A germ $f \in \int \mathfrak{g}$ is called a transversal $A_{1}$ singularity along $\Sigma$ on $X$ if its singular locus $\Sigma_{f}=\Sigma$, and, for $P \in \Sigma \backslash 0, f$ has only $A_{1}$ singularity transversal to the branch of $\Sigma$ containing $P$. It was proved in [3], that $f$ is a transversal $A_{1}$ singularity along $\Sigma$ on $X$ if and only if the Jacobian number $j(f):=\operatorname{dim}\left(\mathfrak{g} /\left(\mathfrak{h}+J_{X}(f)\right)<\infty\right.$.

There exist admissible linear forms $l$ (see [6]) such that $\{l=0\} \cap \Sigma=\{0\}$ and $\{l=0\}$ intersects both $X$ and $\Sigma$ transversally, and $\{l=0\} \cap X \cap f^{-1}(0)$ has isolated singularity at the origin. We assume $\{l=0\}$ is the first coordinate hyperplane $\left\{z_{0}=0\right\}$ of $\mathbb{C}^{n+1}$. 
Let $D_{X}$ be generated by $\xi^{0}, \xi^{1}, \ldots \xi^{s}$. Denote by $D_{X}^{0}$ the submodule of $D_{X}$ generated by those $\xi^{i}$ such that if we write $\xi^{i}=\sum_{j=0}^{n} \xi_{j}^{i} \frac{\partial}{\partial z_{j}}$, then $\xi_{0}^{i} \notin \mathfrak{g}$, and by $D_{X}^{1}$ the submodule of $D_{X}$ generated by those $\xi^{i}$ such that if we write $\xi^{i}=\sum_{j=0}^{n} \xi_{j}^{i} \frac{\partial}{\partial z_{j}}$, then $\xi_{0} \in \mathfrak{g}$. Thus $D_{X}=D_{X}^{0}+D_{X}^{1}$. Denote

$$
J^{0}(f):=D_{X}^{0}(f)=\left\{\xi(f) \mid \xi \in D_{X}^{0}\right\}, \quad J^{1}(f):=D_{X}^{1}(f)=\left\{\xi(f) \mid \xi \in D_{X}^{1}\right\}
$$

If $f$ is clear from the context we just write $J^{0}$ and $J^{1}$.

LEMMA 3. Let $f \in \int \mathfrak{g}$, and $z_{0}, z_{1}, \ldots, z_{n}$ be the coordinates of $\mathbb{C}^{n+1}$ such that $z_{0}=0$ is admissible. The transversal singularity type of $f$ along every branch of $\Sigma$ is constant at all the points of $\Sigma \backslash\{0\}$ if and only if

$$
\operatorname{dim}_{\mathbb{C}}\left(\frac{\mathcal{O}}{\mathfrak{g}+\left(\left(J^{1}+\mathfrak{h}\right): J^{0}\right)}\right)<\infty .
$$

PROOF: The inequality means that $\Sigma \cap \mathcal{V}\left(\left(J^{1}+\mathfrak{h}\right): J^{0}\right)=\{0\}$. For $\varepsilon>0$ small enough, let $P \in \Sigma \cap\left\{z_{0}=t\right\}, 0<|t|<\varepsilon . P \notin \mathcal{V}\left(\left(J^{1}+\mathfrak{h}\right): J^{0}\right)$ if and only if $\left(J^{0}\right)_{P} \subset$ $\left(J^{1}\right)_{P}$, where $\left(J^{i}\right)_{P}$ is the localisation of the ideal at $P$. Since $z_{0}=0$ intersects both $\Sigma$ and $X$ transversally and $X$ is smooth at $P$, we can choose the local coordinates such that locally the branch of $\Sigma$ containing $P$ is the first axis. Furthermore, we can arrange the coordinate transformation such that under this transformation $D_{X}^{0}$ and $D_{X}^{1}$ are preserved. By this we mean that any derivation of $X$ with the first component non-zero at $P$ will remain non-zero at $P$ and any derivation of $X$ with first component zero at $P$ will remain zero at $P$. We let $x=z_{0}, y_{1}, \ldots, y_{n-p}$ be the new local coordinates in a neighbourhood of $P$ in $X$, then at $P,\left(J^{0}\right)_{P}=\left(\frac{\partial f}{\partial x}\right) \mathcal{O}_{X, P}$ and $\left(J^{1}\right)_{P}=\left(\frac{\partial f}{\partial y_{1}}, \ldots, \frac{\partial f}{\partial y_{n-p}}\right) \mathcal{O}_{X, P}$. By [9], the inequality is equivalent to the constancy of the transversal singularity type of $f$.

Let $X, \Sigma$ and $f \in \int \mathfrak{g}$ be the same as before. There is an integer $k_{0}$ such that for all $k \geqslant k_{0}, f_{k}=f+(1 /(k+1)) x^{k+1}$ defines an isolated singularity at $O$. Let $\mu\left(f_{k}\right)$ be the Milnor number of $f_{k}$. If $X$ is a weighted homogeneous complete intersection with isolated singularity, by $[\mathbf{1}, 7.7]$, we have

$$
\mu\left(f_{k}\right)=\operatorname{dim}\left(\frac{\mathcal{O}}{\mathfrak{h}+J_{X}\left(f_{k}\right)}\right) .
$$

By the exact sequence

$$
0 \rightarrow \frac{\mathfrak{g}+J_{X}\left(f_{k}\right)}{\mathfrak{h}+J_{X}\left(f_{k}\right)} \rightarrow \frac{\mathcal{O}}{\mathfrak{h}+J_{X}\left(f_{k}\right)} \rightarrow \frac{\mathcal{O}}{\mathfrak{g}+J_{X}\left(f_{k}\right)} \rightarrow 0
$$

we know that

$$
\mu\left(f_{k}\right)=\operatorname{dim}\left(\frac{\mathfrak{g}+J_{X}\left(f_{k}\right)}{\mathfrak{h}+J_{X}\left(f_{k}\right)}\right)+\operatorname{dim}\left(\frac{\mathcal{O}}{\mathfrak{g}+J_{X}\left(f_{k}\right)}\right)
$$


Define

$$
e_{k}:=\operatorname{dim} \frac{\mathcal{O}}{\mathfrak{g}+J_{X}\left(f_{k}\right)}, \quad \sigma(\Sigma X, 0):=\operatorname{dim}\left(\frac{\mathcal{O}_{\Sigma}}{J_{X}(x)}\right), \quad \operatorname{multi}_{x}(\Sigma):=\operatorname{dim}\left(\frac{\mathcal{O}_{\Sigma}}{(x)}\right) .
$$

Then obviously $e_{k}=\sigma(\Sigma X, 0)+k \operatorname{multi}_{x}(\Sigma)$, and

$$
\frac{\mathfrak{g}+J_{X}\left(f_{k}\right)}{\mathfrak{h}+J_{X}\left(f_{k}\right)}=\frac{\mathfrak{g}}{\left(\mathfrak{h}+J_{X}\left(f_{k}\right)\right) \cap \mathfrak{g}} .
$$

Hence

$$
\mu\left(f_{k}\right)=\sigma(\Sigma X, 0)+k \operatorname{multi}_{x}(\Sigma)+\operatorname{dim}\left(\frac{\mathfrak{g}}{\left(\mathfrak{h}+J_{X}\left(f_{k}\right)\right) \cap \mathfrak{g}}\right)
$$

\section{LiNE SINGULARITIES}

In this section, we assume that $\Sigma$ is a line in $\mathbb{C}^{n+1}$ defined by the ideal $\mathfrak{g}$, and $X$ is a space with isolated complete intersection singularity defined by $\mathfrak{h} \subset \mathfrak{g}$.

LEMMA 4. Let $X$ be a space with isolated complete intersection singularity, containing a line $\Sigma$, which is chosen to be the first axis of a local coordinate system of $\mathbb{C}^{n+1}$. If $X$ is weighted homogeneous with respect to this coordinate system, then $D_{X}=\mathcal{O} \xi_{E}+D_{X}^{1}$ and

$$
\begin{aligned}
J_{X}\left(f_{k}\right) & =\left(\xi_{E}\left(f_{k}\right)\right) \mathcal{O}+J^{1}\left(f_{k}\right) \\
& \subset\left(\xi_{E}\left(f_{k}\right)\right) \mathcal{O}+D_{X}^{1}(f)+D_{X}^{1}\left(x^{k+1}\right),
\end{aligned}
$$

where $J^{1}\left(f_{k}\right)=\left\{\xi\left(f_{k}\right) \mid \xi \in D_{X}^{1}\right\} \subset \boldsymbol{g}$.

PROOF: Let $x, y_{1}, \ldots, y_{n}$ be the local coordinates of $\left(\mathbb{C}^{n+1}, 0\right)$ in the statement of the lemma. We know by [13] that the Euler derivation $\xi_{E}=w_{0} x \frac{\partial}{\partial x}+\sum_{k=1}^{n} w_{k} y_{k} \frac{\partial}{\partial y_{k}} \in D_{X}$, where $w_{0}, w_{1}, \ldots, w_{n}$ are the weights of the coordinates $x, y_{1}, \ldots, y_{n}$ respectively. Let $\xi=\xi^{0} \frac{\partial}{\partial x}+\sum_{k=1}^{n} \xi^{k} \frac{\partial}{\partial y_{k}} \in D_{X}$. Since $\mathcal{O}_{\Sigma}$ is a principal ideal domain and $X$ has isolated singularity at $O, \bar{\xi}^{0} \in(\bar{x})$. Let $\bar{\xi}^{0}=\bar{x} \bar{\xi}_{1}^{0}$. Then $\xi^{\prime}=\xi-\left(1 / w_{0}\right) \xi_{1}^{0} \xi_{E}$ has the coefficient of $\frac{\partial}{\partial x}$ in $\mathbf{g}$.

LEMmA 5. Let $\Sigma$ and $X$ be the same as in Lemma 4. Let $f \in \int \mathfrak{g}$ have transversal $A_{1}$ singularity along $\Sigma$. For $k>0$ sufficiently large, we have

$$
\tilde{J}:=\xi_{E}(f) \mathfrak{g}+D_{X}^{1}(f)+\mathfrak{h}=\left(\mathfrak{h}+J_{X}\left(f_{k}\right)\right) \cap \mathfrak{g}
$$

Proof: By Lemma 4, we have

$$
\left(\mathfrak{h}+J_{X}\left(f_{k}\right)\right) \cap \mathfrak{g}=\left(\xi_{E}\left(f_{k}\right)\right) \cap \mathfrak{g}+D_{X}^{1}\left(f_{k}\right)+\mathfrak{h} .
$$


For $a \in\left(\xi_{E}\left(f_{k}\right)\right) \cap \mathfrak{g}, a=a_{0} x^{k+1}+a_{0} \xi_{E}(f) \in \mathfrak{g}$. Since $\xi_{E}(f) \in \mathfrak{g}, a_{0} \in \mathfrak{g}$. Hence $\left(\xi_{E}\left(f_{k}\right)\right) \cap \mathfrak{g}=\left(\xi_{E}\left(f_{k}\right)\right) \mathfrak{g}$ and

$$
\begin{aligned}
\left(\mathfrak{h}+J_{X}\left(f_{k}\right)\right) \cap \mathfrak{g} & =\left(x^{k+1}+\xi_{E}(f)\right) \mathfrak{g}+D_{X}^{1}\left(f_{k}\right)+\mathfrak{h} \\
& \subset\left(x^{k+1}+\xi_{E}(f)\right) \mathfrak{g}+x^{k} D_{X}^{1}(x)+D_{X}^{1}(f)+\mathfrak{h} .
\end{aligned}
$$

Since $j(f)<\infty$, there is a $k_{1}$ such that when $k>k_{1}, x^{k} \mathfrak{g} \subset \mathfrak{h}+J_{X}(f)=\left(\xi_{E}(f)\right)+$ $D_{X}^{1}(f)+\mathfrak{h}$.

By Lemma 3, there is a $k_{2}$ such that $x^{k_{2}} \in \mathfrak{g}+\left(\left(D_{X}^{1}(f)+\mathfrak{h}\right):\left(\xi_{E}(f)\right)\right)$, and $x^{k_{2}} \xi_{E}(f) \in\left(\xi_{E}(f)\right) \mathfrak{g}+D_{X}^{1}(f)+\mathfrak{h}$. Hence when $k>k_{1}+k_{2}$,

$$
a_{0}\left(x^{k+1}+\xi_{E}(f)\right)+b_{0} x^{k} \in \tilde{J} \text { and }\left(\mathfrak{h}+J_{X}\left(f_{k}\right)\right) \cap \mathfrak{g} \subset \tilde{J} .
$$

On the other hand, there is an integer $n_{1}>0$ such that when $k \geqslant n_{1}$ (see (3.2))

$$
x^{k} \mathfrak{g} \subset\left(\mathfrak{h}+J_{X}\left(f_{k}\right)\right) \cap \mathfrak{g} \subset \tilde{J} \subset \mathfrak{g} .
$$

Then for $k>n_{1}$, by the equality in (4.1)

$$
\begin{aligned}
\tilde{J} & \subset \xi_{E}\left(f+\frac{x^{k+1}}{k+1}\right) \mathfrak{g}+x^{k+1} \mathfrak{g}+D_{X}^{1}(f)+\mathfrak{h} \\
& =\left(\xi_{E}(f)+x^{k+1}\right) \mathfrak{g}+D_{X}^{1}(f)+\mathfrak{h}+x^{k+1} \mathfrak{g} \\
& =\left(\mathfrak{h}+J_{X}\left(f_{k}\right)\right) \cap \mathfrak{g}+x^{k+1} \mathfrak{g} \\
& \subset\left(\mathfrak{h}+J_{X}\left(f_{k}\right)\right) \cap \mathfrak{g}+x^{k} \mathfrak{g} \\
& \subset\left(\mathfrak{h}+J_{X}\left(f_{k}\right)\right) \cap \mathfrak{g}+x^{k-n_{1}} \tilde{J} \\
& \subset\left(\mathfrak{h}+J_{X}\left(f_{k}\right)\right) \cap \mathfrak{g}+\mathfrak{m} \tilde{J} .
\end{aligned}
$$

By Nakayama's lemma, $\left(\mathfrak{h}+J_{X}\left(f_{k}\right)\right) \cap \mathfrak{g}=\tilde{J}$.

LEMMA 6. Under the assumption of Lemma 5, we have

$$
\begin{gathered}
L:=\frac{\mathfrak{h}+J_{X}(f)}{\widetilde{J}} \cong \frac{\left(\xi_{E}(f)\right)}{\left(\xi_{E}(f)\right) \cap \widetilde{J}} \\
\operatorname{Ann}(L)=\mathfrak{g}+\left(\left(D_{X}^{1}(f)+\mathfrak{h}\right):\left(\xi_{E}(f)\right)\right) \\
L \cong \frac{\mathcal{O}}{\operatorname{Ann}(L)}=\frac{\mathcal{O}}{\mathfrak{g}+\left(\left(D_{X}^{1}(f)+\mathfrak{h}\right):\left(\xi_{E}(f)\right)\right)} .
\end{gathered}
$$

Proof: This is an easy exercise in commutative algebra; we omit it.

From the exact sequence

$$
0 \rightarrow \frac{\mathfrak{h}+J_{X}(f)}{\left(\mathfrak{h}+J_{X}\left(f_{k}\right)\right) \cap \mathfrak{g}} \rightarrow \frac{\mathfrak{g}}{\left(\mathfrak{h}+J_{X}\left(f_{k}\right)\right) \cap \mathfrak{g}} \rightarrow \frac{\mathfrak{g}}{\mathfrak{h}+J_{X}(f)} \rightarrow 0
$$


we have

$$
\mu\left(f_{k}\right)=j(f)+e_{k}+\operatorname{dim}\left(\frac{\mathfrak{h}+J_{X}(f)}{\left(\mathfrak{h}+J_{X}\left(f_{k}\right)\right) \cap \mathfrak{g}}\right) .
$$

Notice that $\sigma(\Sigma X, 0)=\operatorname{multi}_{x}(\Sigma)=1, e_{k}=k+1$. By Lemma 6 , we have

$$
\mu\left(f_{k}\right)=k+1+j(f)+\operatorname{dim}\left(\frac{\mathcal{O}}{\mathfrak{g}+\left(\left(D_{X}^{1}(f)+\mathfrak{h}\right):\left(\xi_{E}(f)\right)\right)}\right)
$$

Let $F$ and $F_{k}$ be the Milnor fibre of $f$ and $f_{k}$ respectively. Iomdin-Lê's formula $[6,12]$, says that

$$
\chi(F)=\chi\left(F_{k}\right)+(-1)^{\operatorname{dim} X}(k+1) .
$$

But $\chi\left(F_{k}\right)=1+(-1)^{\operatorname{dim} X-1} \mu\left(f_{k}\right)$. Since in our case $\sigma(\Sigma X, 0)=1$, we have proved

Proposition 7. Let $X$ be a space with isolated complete intersection singularity containing a line $\Sigma$, which is chosen to be the first axis of a local coordinate system of $\mathbb{C}^{n+1}$. Assume that $X$ is weighted homogeneous with respect to the coordinate system. For an analytic function $f \in \int_{X} \mathfrak{g}$ with $j(f)<\infty$, the Euler characteristic of the Milnor fibre $F$ of $f$ is

$$
\chi(F)=1+(-1)^{\operatorname{dim} X-1}(j(f)+\nu)
$$

where

$$
\nu=\operatorname{dim}\left(\frac{\mathcal{O}}{\mathfrak{g}+\left(\left(D_{X}^{1}(f)+\mathfrak{h}\right):\left(\xi_{E}(f)\right)\right)}\right) .
$$

REMARK 8. Formula (4.2) allows us to use a computer program to compute the Euler characteristic effectively. In fact, we have a small Singular [2] program to calculate $\chi(F)$ for a function with critical locus a line on a hypersurface $X$. We used it to check the examples in Example 10.

Let $\Sigma$ be a line in $\mathbb{C}^{n+1}$ defined by the ideal $\mathfrak{g}=\left(y_{1}, \ldots, y_{n}\right), X$ a space with isolated complete intersection singularity defined by $\mathfrak{h}=\left(h_{1}, \ldots, h_{p}\right) \subset \mathfrak{g}$. By changing the generators of $\mathfrak{g}$, we can write

$$
h_{i} \equiv \sum_{k=1}^{p} b_{i k} y_{k} \quad \bmod \mathfrak{g}^{2}
$$

such that the determinant $b$ of the matrix $B=\left(b_{i k}\right)$ is a non-zero divisor in $\mathcal{O}_{\Sigma}$. Note that $y_{1}, \ldots y_{p}$ are projected to zero or the generators of the torsion part $T(M)$ of the conormal module $M=\mathfrak{g} /\left(\mathfrak{g}^{2}+\mathfrak{h}\right)$ of $\mathfrak{g} / \mathfrak{h}$, and $y_{p+1}, \ldots y_{n}$ form a basis of the free module $N=M / T(M)$. We call $\lambda(\Sigma X):=\operatorname{dim}_{\mathbb{C}} T(M)=\operatorname{dim} \mathcal{O} /(\mathfrak{g}+(b))$ the torsion number of the space pair $(\Sigma, X)$. For $f=\sum h_{k l} y_{k} y_{l} \in \mathfrak{g}^{2}$, define

$$
\Delta=\operatorname{det}\left(h_{k l}\right)_{p+1 \leqslant k, l \leqslant n}, \quad \delta_{f}:=\operatorname{dim}\left(\frac{\mathcal{O}}{\mathfrak{g}+\Delta}\right) .
$$


Question 9. For $f \in \mathfrak{g}^{2}$, does the equality $\nu=2 \lambda(\Sigma X)+\delta_{f}-1$ always hold? Or under what conditions does it hold?

The equality in Question 9 and (4.2) show the geometric meaning of $\chi(F)$. By using Singular [2] we have checked that it holds for all the examples we know.

EXAmple 10. Let $X_{k, l}$ be an $A_{k, l}$ singularity defined by $h=x^{l} y+x^{s} z^{2}+y z$. This is an $A_{k}$ singularity with $k=2 l+s-1(l \geqslant 1, s \geqslant 0)$. Since we take the line $\Sigma$ with torsion number $l$ as $x$-axis, we have the definition equation (see [4]). By resolution of singularities, one can prove that for any function $f$ on $X_{k, l}$, if it has isolated line singularity and $A_{1}$ type transversal singularity, then the Milnor fibre $F$ of $f$ is a bouquet of circles (see [11]). Then $\mu(f)=j(f)+\nu$.

(1) We consider a function $g: X_{k, l} \rightarrow \mathbb{C}$. For generic $(a, b, c) \in \mathbb{C}^{3}$, let $g=a y^{2}+b y z+c z^{2}$, for example, take $g=y^{2}-y z+z^{2} / 2$. A calculation shows that $\mu(g)=6 l-3$.

(2) Let $f: X_{k, l} \longrightarrow \mathbb{C}$ be defined by $f=y+z^{2} / 2$. A calculation shows that

$$
\mu(f)= \begin{cases}l-1 & \text { if } s=0 \\ l+3 s-2 & \text { if } 1 \leqslant s \leqslant l-1 \\ 4 l-2 & \text { if } s \geqslant l .\end{cases}
$$

\section{REFERENCES}

[1] J.W. Bruce and R.M. Roberts, 'Critical points of functions on analytic varieties', Topology 27 (1988), 57-90.

[2] G.-M. Greuel, G. Pfister and H. Schoenemann, 'Singular', (A computer algebra system, ftp:helios@mathematik.uni-kl.de or http://www.mathematik.uni-kl.de).

[3] G. Jiang, Functions with non-isolated singularities on singular spaces, Thesis (Utrecht University, 1998).

[4] G. Jiang, D. Siersma, 'Local embeddings of lines in singular hypersurfaces', Ann. Inst. Fourier (Grenoble) 49 (1999), 1129-1147.

[5] G. Jiang and A. Simis, 'Higher relative primitive ideals', Proc. Amer. Math. Soc. (to appear).

[6] D.T. Lê, 'Ensembles analytiques complex avec lieu singulier de dimension un (d'après I.N. Iomdin)', Publ. Math. Univ. Paris VII (1980), 87-94.

[7] R. Pellikaan, 'Finite determinacy of functions with non-isolated singularities', Proc. London Math. Soc. 57 (1988), 357-382.

[8] R. Pellikaan, Hypersurface singularities and resolutions of Jacobi modules, Thesis (Rijksuniversiteit Utrecht, 1985).

[9] R. Pellikaan, 'Series of isolated singularities', Contemp. Math. 90 (1989), 241-259.

[10] K. Saito, 'Theory of logarithmic differential forms and logarithmic vector fields', J. Fac. Sci. Univ. Tokyo Sect $1 A$ Math. 27 (1980), 265-291.

[11] P. Seibt, 'Differential filtrations and symbolic powers of regular primes', Math. Z. 166 (1979), 159-164. 
[12] M. Tibăr, 'Embedding non-isolated singularities into isolated singularities', in Singularities, the Brieskorn anniversary volume, (V.I. Arnold, G.-M. Greuel, J.H.M. Steenbrink, Editors), Progress in Math. 162 (Birkhäuser Verlag, Boston MA, 1998), pp. 103-115.

[13] J.M. Wahl, 'Derivations, automorphisms and deformations of quasi-homogeneous singularities', in Singularities, Part 2 (Arcata, Calif., 1981), Proc. Sympos. Pure Math. 40 (Amer. Math. Soc., Providence, R.I., 1983), pp. 613-624.

\author{
Department of Mathematics \\ Jinzhou Normal University \\ Jinzhou City \\ Liaoning 121000 \\ Peoples Republic of China \\ e-mail: jzjgf@public2.jzptt.ln.cn
}

